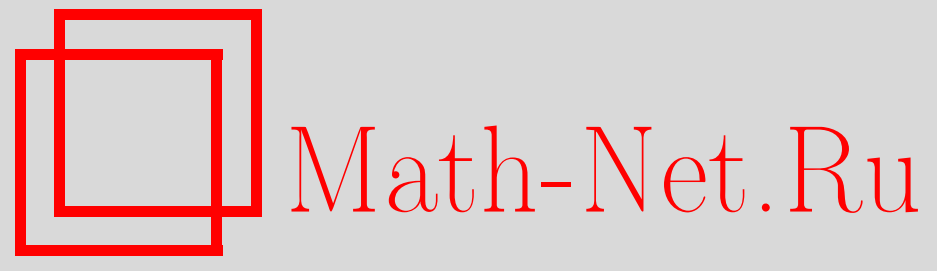

Ю. М. Лифшиц, Разбиения $k$-связного графа на части, Дискрет. матем., 2005, том 17, выпуск 3, 112-122

DOI: https://doi.org/10.4213/dm121

Использование Общероссийского математического портала Math-Net.Ru подразумевает, что вы прочитали и согласны с пользовательским соглашением http://www.mathnet.ru/rus/agreement

Параметры загрузки:

IP: 3.82 .47 .9

26 апреля 2023 г., 18:16:51

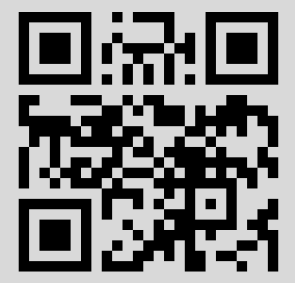


Удк 519.6

\title{
Разбиения $k$-связного графа на части
}

\author{
() 2005 г. .М.Лифшиц
}

В статье изучается разбиение графа набором разделяющих множеств. Найдена точная верхняя оценка числа получающихся частей. Исследована структура набора в случае достижения этой оценки. Кроме того, результат Д. В. Карпова о числе частей в разбиении передоказан при более слабых предположениях о графе. В статье также доказана теорема о пограничных частях, дающая верхнюю оценку числа частей разбиения, граничащих с данной вершиной.

Работа выполнена при поддержке программы фундаментальных исследований Президиума РАН «Исследования в основных областях современной математики» и программы Президента Российской Федерации поддержки ведущих научных школ, грант НШ-2203.2003.1.

\section{1. Введение}

При изучении связности графа встает естественный вопрос, как выделить в $k$-связном графе подграфы большей связности и какую систему они образуют. В связном графе максимальное по включению подмножество вершин, индуцирующее двусвязный подграф, называется блоком. Структура блоков исследована достаточно полно (см., например, $[1,2])$. В работах $[3,4]$ были предприняты попытки обобщить основные результаты этой теории на графы большей связности. Ряд работ был посвящен трехсвязным блокам в двусвязных графах [5, 6, 7]. В [8], а затем и [9] вводятся новые методы разбиения $k$-связного графа на $(k+1)$-связные блоки. В [10] появляется понятие части разбиения графа по набору разделяющих множеств. Это понятие тесно связано с построением блоков в $k$-связном графе. В частности, любой блок $k$-связного графа можно представить как часть разбиения этого графа всеми $k$-разделяющими множествами. Кроме того, в [10] при довольно сильных ограничениях на разделяющий набор и сам граф был получен следующий результат: набор из $n$ разделяющих множеств делит граф не более чем на $2 n$ частей.

Статья построена следующим образом. Сначала мы введем основные понятия теории $k$-связных графов и приведем их простейшие свойства, которые понадобятся при доказательстве основных результатов. Затем мы докажем теорему о пограничных частях, которая будет основным инструментом для получения оценок числа частей в разбиении. После этого будет получена следующая теорема о полном разбиении. Пусть в некотором $k$-связном графе есть набор $k$-разделяющих множеств $\left\{A_{1}, \ldots A_{n}\right\}$. Для каждого $1 \leqslant i \leqslant n$ обозначим через $a_{i}$ число частей, на которые множество $A_{i}$ делит граф. Тогда весь набор делит граф не более чем на

$$
\sum_{i=1}^{n} a_{i}-\sum_{i=1}^{n}\left[a_{i}>2\right]+\left[\forall i a_{i}>2\right]
$$


невырожденных частей. В этой формуле [утверждение] - это нотация Айверсона (см. $[11,12])$, то есть результат этого выражения равен 1 , если утверждение в квадратных скобках верно, и равен 0 в противном случае. Кроме того, будет показано, что результат Д. В. Каргова из [10] о $2 n$ частях является частным случаем этой теоремы. В следующем разделе будет описана структура разделяющего набора в случае полного разбиения, то есть в случае, когда число частей в разбиении графа достигает полученной нами верхней оценки. В последнем разделе мы докажем, что число невырожденных частей в разбиении графа $n$ разделяющими множествами не превосходит $2 n$ при более слабых ограничениях на граф, чем в [10].

\section{2. Основные понятия}

Определение 1. Назовем простой (без петель и кратных ребер) граф $k$-связным, если при удалении любой $k-1$ вершины (и всех ребер, выходящих из этих вершин) граф остается связным.

Определение 2. Назовем подмножество вершин $A$ графа разделяющим, если при удалении этих вершин граф перестает быть связным. Само удаление назовем разрезом графа по $A$ (или $A$-разрезом). Соответственно, назовем подмножество вершин графа $m$-разделяющим, если оно разделяющее и состоит из $m$ вершин.

В этой статье речь будет идти только о $k$-разделяющих множествах в $k$-связном графе.

Определение 3. Будем говорить, что разделяющее множество $A$ разделяет набор вершин $X$, если при $A$-разрезе найдутся две неудаленные вершины $X$, попавшие в разные компоненты связности. Будем говорить, что $A$ отделяет вершину $x \notin A$ от множества $P$, если при $A$-разрезе найдется неудаленная вершина из $P$, попавшая не в ту компоненту связности, в которой оказалась $x$.

Теперь определим понятие части и укажем его основное свойство.

Определение 4. Пусть $\mathfrak{U}$ - набор разделяющих множеств графа $G$. Множество $P$ из нескольких вершин графа назовем частью $\mathfrak{A}$-разбиения, если никакие две вершины из $P$ нельзя разделить никаким множеством из набора $\mathfrak{U}$, но любая другая вершина графа может быть отделена от множества $P$ разрезом по одному из множеств $\mathfrak{U}$.

В дальнейшем слово разбиение всегда будет относиться к набору разделяющих множеств, а разрез - к одному разделяющему множеству.

Пусть набор $\mathfrak{U}$ состоит только из одного разделяющего множества $A$, и пусть при $A$-разрезе граф распадается на компоненты связности $F_{1}, \ldots, F_{l}$. Тогда согласно определению частями $\mathfrak{Q}$-разбиения ( $A$-разреза) будут множества $A \cup F_{1}, \ldots, A \cup F_{l}$.

Необходимо отметить, что если рассмотреть часть как отдельный граф (проведя между вершинами части те ребра, которые были в исходном графе), то он может оказаться несвязным.

Пример 1. Рассмотрим простой цикл $t u v x y z$, являющийся двухсвязным графом, представленный на рис. 1. Тогда $A=\{u, z\}$ и $B=\{v, y\}$ - его 2-разделяющие множества. В $\{A, B\}$-разбиении три части: $\{t, u, z\},\{u, v, y, z\}$ и $\{v, x, y\}$. 


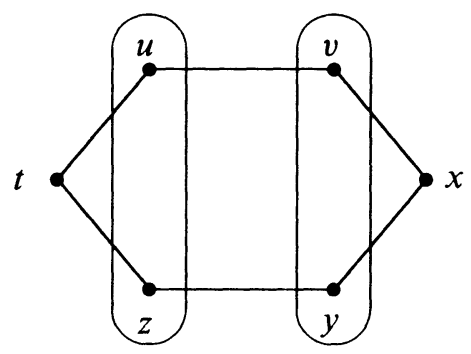

Puc. 1.

Предложение 1. Если $Q$ - часть $\mathfrak{U}-$ разбиения, то ее можно представить в виде

$$
Q=\bigcap_{A \in \mathfrak{X}} P_{A}
$$

где $P_{A}$ - одна из частей А-разреза графа.

Возьмем по одной части разреза по каждому из множеств $\mathfrak{U}$. Все такие выборки будем называть $\mathfrak{U}$-комбинациями или просто комбинациями. Тогда, другими словами, предложение 1 говорит, что любая часть $\mathfrak{U}$-разбиения является пересечением частей какой-то комбинации. Доказательство предложения 1 несложно и приведено в [10] (см. теорему 1 в [10]). Таким образом, общее число частей в $\mathscr{U}$-разбиении не превосходит произведения чисел частей в разрезах по каждому разделяющему множеству из $\mathfrak{U}$.

Теперь введем понятия границы и внутренности части, оперируя с которыми мы сможем получить основные результаты этой статьи.

Определение 5. Границей части $\mathscr{U}$-разбиения $P$ назовем множество тех вершин $P$, которые лежат хотя бы в одном из множеств $\mathfrak{A}$, а размером границы - их число. Соответственно, внутренностью части назовем множество остальных вершины $P$.

Определение 6. Часть $\mathfrak{Q}$-разбиения назовем вырожденной, если ее внутренность пуста.

Вырожденная часть разбиения не обязательно является пустым множеством. В следующем предложении дается основное свойство границы.

Предложение 2. Если внутренность части не пуста, то ее граница является разделяюцим множеством (не обязательно ровно из $k$ вершин), отделяющим эту внутренность от остальных вериин графа.

Это также нетрудно проверить (см. замечание к определению 4 в [10]). Таким образом, для любого набора $\mathfrak{U}$ разделяющих множеств в $k$-связном графе граница любой невырожденной части $\mathfrak{X}$-разбиения состоит хотя бы из $k$ вершин.

Теперь приведем последнее утверждение (нетрудное доказательство опустим), которое нам потребуется.

Предложение 3. Пусть

$$
Q=\bigcap_{A \in \mathfrak{A}} P_{\boldsymbol{A}},
$$


где $P_{A}$ - одна из частей $A$-разреза графа. Тогда если $Q$ содержит хотя бы одну вершину, не лежашую ни в одном из множеств $\mathfrak{X}$, то $Q-$ часть $\mathfrak{X}-$ разбиения, причем невырожденная.

Другими словами, если пересечение частей какой-то комбинации содержит хотя бы одну вершину не из множеств $\mathfrak{A}$, то оно является частью разбиения, причем невырожденной.

\section{3. Теорема о пограничных частях}

Рассмотрим произвольную вершину $y$ нашего графа. Для каждого разделяющего множества из $\mathfrak{U}$, содержащего $y$, возьмем число частей, на которое оно режет граф. Сумму взятых чисел назовем весом вершины $y$ относительно $\mathfrak{U}$.

Теорема 1. Число невырожденных частей $\mathfrak{2}$-разбиения, с которыми граничит (то есть входит в граничу) произвольная вериина графа, не превосходит веса этой вериины относительно набора $\mathfrak{A}$.

Доказательство. Пусть $\mathfrak{U}=\left\{A_{1}, \ldots, A_{n}\right\}$, и $A_{i}$ режет граф на $a_{i}$ частей, $1 \leqslant i \leqslant n$. Пусть вершина $x$ принадлежит множествам $A_{1}, \ldots, A_{m}$ и не принадлежит остальным. Тогда нам нужно доказать, что эта вершина является граничной не более чем для $\sum_{i=1}^{m} a_{i}$ невырожденных частей $\mathfrak{U}$-разбиения. Иными словами вес вершины $y$ равен $\sum_{i=1}^{n} a_{i}\left[y \in A_{i}\right]$.

Таким образом, в утверждении теоремы неявно присутствуют два параметра, $m$ и $n$. Будем проводить доказательство индукцией по $m$ сразу при всех $n$.

Рассмотрим базу индукции при $m=1$. Пусть множество $A$ из набора $\mathfrak{X}$ делит граф на $a$ частей. Вершина $x$ принадлежит $A$ и не принадлежит остальным множествам $\mathfrak{U}$. Будем рассматривать части $\mathfrak{2}$-разбиения в виде, представленном в предложении 1. Заметим, что вершина $x$ принадлежит всем $a$ частям $A$-разреза, а для разрезов по каждому из остальных разделяющих множеств набора $\mathfrak{U}$ вершина $x$ принадлежит только какой-то одной части. Таким образом, $x$ принадлежит пересечению только $a$ комбинаций. Следовательно, $x$ принадлежит (а значит и является граничной) не более чем $a$ частям.

Рассмотрим индуктивный переход $(m-1) \rightarrow m$. По построению $n \geqslant m$. Разберем сначала случай $n=m$, то есть случай, когда $x$ принадлежит всем множествам $\mathfrak{U}$. Будем доказывать, что во всем $\mathfrak{A}$-разбиении не более, чем $a=\sum_{i=1}^{n} a_{i}$ невырожденных частей, от противного. Предположим, что этих частей $a+b$, где $b>0$. Обозначим через $C$ объединение всех множеств $\mathfrak{U}$ и через $D$ их пересечение. Пусть $d=|D|$. Оценим сумму размеров границ невырожденных частей $\mathfrak{U}$-разбиения (обозначим это число через $T$ ) двумя способами.

По индукционному предположению для каждой вершины из $C \backslash D$ число частей, в границу которых она входит, не превосходит ее веса. Каждая вершина из $D$ граничит не больше чем с $a+b$ невырожденными частями. Вес каждой вершины из $D$ равен $a$. Таким образом, сумма размеров границ невырожденных частей $\mathfrak{U}$-разбиения превышает общий вес всех вершин не более чем на $d b$. Сумма весов всех вершин равна

$$
k \sum_{i=1}^{n} a_{i}=k a,
$$

так как каждое множество $A_{i}$ вносит в суммарный вес вершин $C$ вклад, равный $k a_{i}$. Итак, $T \leqslant k a+d b$. 
С другой стороны, как уже было отмечено в предложении 2, граница каждой невырожденной части состоит не менее чем из $k$ вершин. Следовательно, $T \geqslant b k+a k$, откуда $d \geqslant k$, что противоречит определению $d$. Таким образом, всего невырожденных частей $\mathfrak{A}$-разбиения не больше $a$, следовательно и $x$ граничит не более чем с $a$ частями.

Разберем теперь случай $n>m$. Применяя теорему к уже доказанному случаю $n=m$, получим, что найдется не более $a=\sum_{i=1}^{m} a_{i}$ невырожденных частей $\mathfrak{B}$-разбиения, $\mathfrak{B}=\left\{A_{1}, \ldots, A_{m}\right\}$. Отсюда, по предложению 3 , число $\mathfrak{B}$-комбинаций, пересечение которых содержит хотя бы одну вершину, не лежащую ни в одном из множеств $\mathfrak{U}$, не более $a$. Как и в рассуждениях при доказательстве базы индукции, вершина $x$ принадлежит только какой-то одной части разреза по каждому из разделяющих множеств набора $\mathfrak{A} \backslash \mathfrak{B}$. Следовательно, уже $\mathfrak{Q}$-комбинаций, пересечение частей которых будет представлять невырожденную часть $\mathfrak{U}$-разбиения, содержащую $x$, будет не больше $a$. Таким образом, $x$ входит в границу не более чем $a$ невырожденных частей.

\section{4. Основная теорема}

Для дальнейших рассуждений нам необходимо подробно изучить разбиение графа двумя разделяющими множествами. Сейчас мы введем набор понятий и обозначений (клеточный язык), которые будут использованы в доказательстве почти всех последующих утверждений.

Пусть $A$ и $B-k$-разделяющие множества, делящие граф соответственно на $a$ и $b$ частей. Обозначим части $A$-разреза через $P_{1}, \ldots, P_{a}$, части $B$-разреза через $Q_{1}, \ldots, Q_{b}$. Это значит, что при удалении множества $A$ из графа он разделится на компоненты связности $P_{1} \backslash A, \ldots, P_{a} \backslash A$.

Опишем таблицу $(a+1) \times(b+1)$. Ее столбцы будут соответствовать множествам $A, P_{1} \backslash A, \ldots, P_{a} \backslash A$, строчки - множествам $B, Q_{1} \backslash B, \ldots, Q_{b} \backslash B$. В каждую клетку поместим вершины графа из пересечения множеств, соответствующих строке и столбцу этой клетки. Клетку будем называть пустой, если в ней не лежит ни одной вершины. В левой верхней клетке (будем называть ее угловой) будут лежать вершины множества $D=A \cap B$. Клетки левого (нулевого) столбца и верхней (нулевой) строчки будем называть крайними, остальные - внутренними. Будем говорить, что внутренняя клетка граничит с самой верхней клеткой своего столбца, с самой левой клеткой своей строки и с угловой клеткой. Число вершин в клетках зададим матрицей $\left\{g_{i j}\right\}$, где $0 \leqslant j \leqslant a, 0 \leqslant i \leqslant b$.

В этих обозначениях предложение 1 можно сформулировать следующим образом: любая невырожденная часть $\{A, B\}$-разбиения есть объединение вершин какой-то непустой внутренней клетки и граничащих с ней крайних клеток. По предложению 3 , каждое такое объединение является невырожденной частью, причем ее границей будут в точности вершины из крайних клеток, ведь это те вершины этой части, которые лежат в $A$ или $B$. Внутренностью этой части будут вершины данной внутренней клетки. Согласно предложению 2 , общее число вершин в клетках, граничащих с данной непустой внутренней клеткой, должно быть не меньше $k$. Это можно также сформулировать следующим образом:

$$
1 \leqslant j \leqslant a, \quad 1 \leqslant i \leqslant b, \quad g_{i j} \neq 0 \quad \Longrightarrow \quad g_{00}+g_{0 j}+g_{i 0} \geqslant k .
$$

Определение 7. Пусть $A$ и $B-k$-разделяющие множества. Назовем часть $B$-разреза $Q$ дальней для $A$, если существует не более одной части $A$-разреза $P$ такой, что $P \cap Q$ содержит хотя бы одну вершину вне $A \cup B$. В противном случае будем называть $Q$ ближней. 
Переведем это определение на клеточный язык: часть $Q_{i}$ дальняя, если в строке $Q_{i} \backslash B$ не более одной непустой внутренней клетки, то есть среди чисел $g_{i 1}, \ldots, g_{i a}$ не более одного ненулевого.

Лемма 1. Пусть $A$ и $B-k$-разделяюшие множества, делящие граф соответственно на a и $b$ частей, $a \geqslant 3$. Тогда среди частей В-разреза есть не более одной ближней для $A$.

Доказательство. Будем доказывать эту лемму на клеточном языке, используя метод от противного. Пусть $Q_{1}$ и $Q_{2}$ - ближние части. Это значит, что в первой и второй строках нашей таблицы есть хотя бы по две непустых внутренних клетки. Отметим по непустой внутренней клетке в этих строках так, чтобы отмеченные клетки лежали в разных столбцах. Не умаляя общности можно считать, что это клетки $(1,1)$ и $(2,2)$. Введем обозначения для крайних клеток, которые граничат с выбранными. Пусть

$$
\begin{array}{ll}
A_{1}=A \cap\left(Q_{1} \backslash B\right), & A_{2}=A \cap\left(Q_{2} \backslash B\right), \\
B_{1}=B \cap\left(P_{1} \backslash A\right), & B_{2}=B \cap\left(P_{2} \backslash A\right),
\end{array}
$$

а также пусть $D=A \cap B$.

Используя предложение 2 в клеточной формулировке, получим, что

$$
g_{00}+g_{01}+g_{10} \geqslant k, \quad g_{00}+g_{02}+g_{20} \geqslant k .
$$

С другой стороны $A$ и $B-k$-разделяющие множества, откуда

$$
g_{00}+g_{10}+g_{20} \leqslant k, \quad g_{00}+g_{01}+g_{02} \leqslant k .
$$

Следовательно,

$$
g_{01}=g_{20}, \quad g_{10}=g_{02}, \quad g_{00}+g_{01}+g_{02}=g_{00}+g_{10}+g_{20}=k .
$$

Вспомним теперь о том, что $a \geqslant 3$. Рассмотрим $P_{3}$, еще одну часть $A$-разреза. Тогда $P_{3} \backslash A-$ вершины одной из компонент связности, остающейся после удаления $A$. Следовательно $P_{3} \backslash A$ (ребра проводим как в исходном графе) - связный граф. Более того, из любой вершины $A$ есть ребро, ведущее в $P_{3} \backslash A$, иначе $A$ не было бы минимальным по числу вершин разделяющим множеством. Из равенств (1) следует, что все вершины $B$ лежат в $P_{1} \cup P_{2}$, следовательно, их нет в $P_{3} \backslash A$. Отсюда получаем, что $P_{3} \backslash A$ лежит в одной компоненте связности после удаления $B$, более того, в этой же компоненте лежат все вершины $A \backslash(A \cap B)$. Последнее утверждение верно, поскольку из каждой вершины $A \backslash(A \cap B)$ выходит ребро, ведущее в $P_{3} \backslash A$. По определению $A_{1}$ состоит из вершин $A$, попавших в первую компоненту $B$-разреза, а $A_{2}$ - из вершин, попавших во вторую. Следовательно, либо $A_{1}$, либо $A_{2}$ пусто. Откуда, $g_{10}=0$ или $g_{20}=0$, не умаляя общности можно считать, что верно второе. Тогда из (1) следует, что

$$
g_{20}=g_{01}=0, \quad g_{10}=g_{02}, \quad g_{00}+g_{10}=g_{00}+g_{02}=k .
$$

Таким образом, все вершины $B$ распределены между $D$ и $B_{2}$. Теперь посмотрим на вторую строчку (то есть $\left.Q_{2} \backslash B\right)$ в свете только что полученной формулы $(2)$. Кроме $(2,2)$ в ней должна быть еще одна непустая внутренняя клетка. Посмотрим на граничащие с ней крайние клетки. Самая левая в строке клетка $A_{2}=A \cap\left(Q_{2} \backslash B\right)$ пустая. Самая верхняя в столбце, это не $D$ и не $B_{2}$, следовательно, тоже пустая. Поэтому граница невырожденной части, соответствующей рассматриваемой внутренней клетке, состоит только из $D$. Но $|D|<k$, и мы приходим к противоречию с предложением 2 в клеточной форме. 
Замечание 1. Почти такими же рассуждениями можно показать, что в условиях только что доказанной леммы среди частей $A$-разреза тоже не более одной ближней для $B$.

Теорема 2. Пусть $\mathfrak{U}=\left\{A_{1}, \ldots A_{n}\right\}$-набор $k$-разделяющих множеств $k$-связного графа. Пусть $a_{i}, 1 \leqslant i \leqslant n,-$ число частей, на которые множество $A_{i}$ режет граф. Тогда весь набор разбивает граф не более чем на

$$
\sum_{i=1}^{n} a_{i}-\sum_{i=1}^{n}\left[a_{i}>2\right]+\left[\forall i \quad a_{i}>2\right]
$$

невырожденных частей.

Доказательство. Будем доказывать теорему индукцией по числу тех $i$, для которых $a_{i}>2$, иначе говоря, индукщией по $\sum_{i=1}^{n}\left[a_{i}>2\right]$.

В качестве базы рассмотрим два случая. В первом случае $n=1, a_{1}>2$. Тогда существует $a_{1}$ невырожденных частей $A_{1}$-разреза и это ровно тот результат, который дает формула (3).

Во втором случае $a_{1}=\ldots=a_{n}=2$. Пусть $C$ - объединение множеств $\mathfrak{A}$. Согласно теореме о пограничных частях число частей, с которыми граничит данная вершина $C$, не превосходит веса этой вершины. Суммарный вес вершин $C$ равен $k \sum_{i=1}^{n} a_{i}$. Размер границы каждой невырожденной части не меньше $k$. Следовательно, число невырожденных частей не больше $\sum_{i=1}^{n} a_{i}=2 n$, формула (3) доказана.

Рассмотрим индукционный переход. Пусть $\mathfrak{U}$ не описывается ни одним из двух только что разобранных случаев. Тогда $n \geqslant 2$ и существует $i$ такое, что $a_{i}>2$. Не умаляя общности, можно считать, что $a_{n}>2$. Применим для $\mathfrak{U}^{\prime}=\mathfrak{U} \backslash\left\{A_{n}\right\}$ индукщионное предположение. Заметим, что формула (3) дает для $\mathfrak{U}$ результат на $a_{n}-1$ больший, чем для $\mathfrak{U}^{\prime}$. Докажем, что число невырожденных частей в $\mathfrak{U}$-разбиении увеличилось не более чем на $a_{n}-1$ по сравнению с $\mathfrak{U}^{\prime}$.

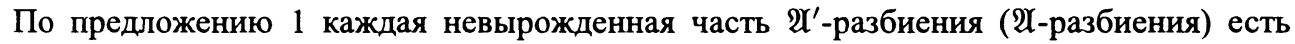
пересечение какой-то $\mathfrak{U}^{\prime}$-комбинации (趾омбинации). Если $\mathfrak{U}$-комбинация получается из данной $\mathfrak{U}^{\prime}$-комбинации добавлением какой-то части $A_{n}$-разреза, будем говорить, что первая выросла из второй. Посмотрим, из чего выросли комбинации, соответствующие невырожденным частям $\mathfrak{U}$-разбиения.

Если $\mathfrak{Q}^{\prime}$-комбинация не соответствует никакой невырожденной части $\mathfrak{U}^{\prime}$-разбиения, то ее пересечение (по предложению 3) содержит только вершины из множеств $\mathfrak{U}^{\prime}$. Следовательно, пересечение частей любой комбинации, которая вырастет из данной, может дать только вырожденную часть $\mathfrak{A}$-разбиения.

Рассмотрим случай, когда $\mathfrak{U}^{\prime}$-комбинация соответствует какой-то невырожденной части $\mathfrak{U}^{\prime}$-разбиения и содержит хотя бы одну дальнюю часть для $A_{n}$. Тогда, по определению дальней части, к этой комбинации можно добавить не более одной части $A_{n}$-разреза так, чтобы пересечение полученной $\mathfrak{U}$-комбинации не лежало целиком в объединении множеств $\mathfrak{A}$. Следовательно, из нашей комбинации вырастет не более одной $\mathfrak{X}$-комбинации, соответствующей невырожденной части $\mathfrak{U}$-разбиения.

Наконец, по лемме 1 у каждого разделяющего множества из набора $\mathfrak{U}^{\prime}$ имеется не более одной ближней части для $A_{n}$. Следовательно, существует не более одной ближней $\mathfrak{U}^{\prime}$-комбинации, соответствующей невырожденной части $\mathfrak{U}^{\prime}$-разбиения и не содержащей ни одной дальней части. Из такой части может вырасти не более $a_{n} \mathfrak{A}$-комбинаций, соответствующих невырожденным частям $\mathfrak{U}$-разбиения. 
Таким образом, число $\mathfrak{U}$-комбинаций, соответствующих невырожденным частям $\mathfrak{A}$-разбиения, превосходит количество невырожденных частей $\mathfrak{U}^{\prime}$-разбиения не более чем на $a_{n}-1$.

Замечание 2. Если $k>1$, то для любого набора $a_{1}, \ldots, a_{n}$ существуют граф и соответствующий этим числам набор $\mathfrak{U}$ разделяющих множеств, для которых число невырожденных частей в разбиении в точности равно (3). Если $k=1$, то можно доказать более сильную оценку: в разбиении связного графа 1-разделяющими множествами будет не более $1-n+\sum_{i=1}^{n} a_{i}$ невырожденных частей. Отличие от основного случая объясняется тем, что если резрез по каждой-либо из двух вершин состоит из двух частей, то разбиение по этой паре не может содержать более трех частей.

Определение 8. Назовем $\mathfrak{A}$-разбиение полным, если оно делит граф на число частей, вычисляемое по формуле (3).

Замечание 3. Из рассуждений в доказательстве теоремы 2 следует, что если $\mathfrak{A}$-разбиение полное, $a_{n}>2$ и $n>1$, то разбиение $\mathfrak{U}^{\prime}=\mathfrak{U} \backslash A_{n}$ тоже должно быть полным.

Как было доказано в [10] (см. теорему 6 в [10]), если на граф и набор наложены специальные условия (граф должен быть слабо нерасщепимым и каждый представитель набора $\mathfrak{U}$ из $n$ множеств должен быть зависим с каким-то разделяющим множеством графа), то в $\mathfrak{U}$-разбиении найдется не более $2 n$ невырожденных частей. Этот результат является частным случаем только что доказанной теоремы 2, так как в силу леммы 6 из [10] при наложенных на граф и набор $\mathfrak{U}$ условиях каждое множество $\mathfrak{U}$ режет граф на две части.

\section{5. Полные разбиения}

Теорема 3. Пусть $\mathfrak{U}$ - полное разбиение, $\mathfrak{U}^{\prime}=\left\{A_{1}, \ldots, A_{k}\right\}-$ все его разделяючие множества, делящие граф на две части (то есть $\left.a_{1}=\ldots=a_{k}=2\right)$. Тогда $\mathfrak{U}^{\prime}$-разбиение тоже полное, то есть в нем $2 k$ частей.

Доказательство. Докажем утверждение индукщией по $n-k$, где $n=|\mathfrak{A}|$. При $n=k$ утверждение очевидно. Проведем шаг индукции. Сначала применим замечание к теореме 2 (то есть выкинем $A_{n}$ из $\mathfrak{U}$ ), затем используем индукционное предположение.

Определение 9. Назовем пару разделяющих множеств $A, B$ независимой, если при $B$-разрезе все неудаленные вершины $A$ оказались в одной компоненте связности. В противном случае будем называть ее зависимой с $B$.

В работах $[8,9]$ показано, что отношение независимости симметрично относительно множеств $A$ и $B$. Далее мы будем просто писать, что $A$ и $B$ независимы (зависимы).

Теорема 4. Пусть набор $\mathfrak{X}$ дает полное разбиение, и пусть одно из множеств этого набора $A$ режет граф на $а>2$ частей. Тогда $A$ независимо со всеми остальными множествами $\mathfrak{U}$.

Доказательство. Возьмем произвольное множество $B$ из $\mathfrak{U}$, отличное от $A$. Будем изучать разбиение графа набором $\{A, B\}$. Пусть $\mathfrak{U}^{\prime}=\mathfrak{U} \backslash A$. Мы будем пользоваться стандартными обозначениями клеточного языка. 
Убедимся, что хотя бы одна из $b$ частей $B$-разреза является ближней к $A$. Если бы все части были дальнимй, то из каждой $\mathfrak{A}^{\prime}$-комбинации (соответствующей невырожденной части) вырастало бы не более одной невырожденной части $\mathfrak{A}$, что противоречит полноте $\mathfrak{U}$-разбиения. Не умаляя общности можно считать, что $Q_{1}$ ближняя. Тогда каждая внутренняя клетка в первой строке (соответствующей $Q_{1} \backslash B$ ) невырождена. Действительно, в противном случае из ближней $\mathfrak{U}^{\prime}$-комбинации вырастет не более $a-1$ невырожденной час-

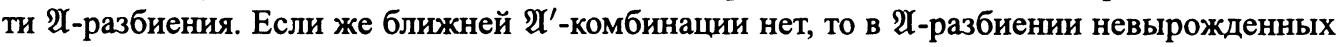
частей будет не больше чем в $\mathfrak{U}^{\prime}$-разбиении. В любом случае, приходим к противоречию с условием полноты.

Докажем еще один необходимый факт, состоящий в том, что в клеточной таблице есть хотя бы одна непустая внутренняя клетка вне первой строки. Рассмотрим $\mathfrak{2}^{\prime \prime}=\mathfrak{Q} \backslash \boldsymbol{B}$. Если бы сформулированный факт не был верен, то из каждой части $\mathfrak{U}^{\prime \prime}$-разбиения выросло бы не более одной невырожденной части $\mathfrak{\mathscr { A }}$-разбиения (во все $\mathfrak{U}^{\prime \prime}$-комбинации пришлось бы добавлять только $Q_{1}$, чтобы получить невырожденную часть). Мы вновь получили противоречие с условием полноты, так как формула (3) показывает, что в $\mathfrak{X}^{\prime \prime}$-разбиении должно быть хотя бы на одну часть меньше. Не умаляя общности, будем считать, что найденная непустая внутренняя клетка есть клетка $(2,1)$.

Теперь рассмотрим, по каким крайним клеткам разошлись вершины $A$ и $B$. Воспользуемся предложением 2 в клеточной форме:

$g_{00}+g_{10}+g_{01} \geqslant k, \quad g_{00}+g_{10}+g_{02} \geqslant k, \quad g_{00}+g_{10}+g_{03} \geqslant k, \quad g_{00}+g_{20}+g_{01} \geqslant k$.

Исходя из размеров $A$ и $B$, получаем, что $g_{00}+g_{10}+g_{20} \leqslant k$ и $g_{00}+g_{01}+g_{02}+g_{03} \leqslant k$. Из этих двух серий неравенств сразу находим, что $g_{00}+g_{10}=k$, следовательно, все вершины $A$ лежат в части $B$-разреза $Q_{1}$, то есть $A$ и $B$ независимы.

\section{6. Оценка числа частей в разрезе графа одним множеством}

Теперь обратимся к вопросу, как оценить число частей в разрезе $k$-связного графа одним разделяющим множеством.

Теорема 5. Пусть $A$ и $B$-зависимые разделяющие множества $k$-связного графа, $p \geqslant 2$, и степени всех вершин из множеств $А$ и В не меньше $k(p+2) /(p+1)$. Тогда каждое из них делит граф не более чем на р частей.

Доказательство. Докажем теорему от противного. Предположим, что число частей в $A$-разрезе не меньше $p+1$. Будем вести рассуждения на клеточном языке. Докажем методом от противного, что в таблице для $A$ и $B$ не может быть двух непустых внутренних клеток, лежащих одновременно и в разных столбцах, и в разных строках. Предположим, что такие клетки нашлись. Не умаляя общности, можно считать, что это клетки $(1,1)$ и $(2,2)$. Тогда по предложению 2 в клеточной форме

$$
g_{00}+g_{01}+g_{10} \geqslant k, \quad g_{00}+g_{02}+g_{20} \geqslant k .
$$

Исходя из размеров $A$ и $B$, получаем, что

$$
g_{00}+g_{10}+g_{20} \leqslant k, \quad g_{00}+g_{01}+g_{02} \leqslant k .
$$


Мы предположили, что $A$ делит граф на $p+1$ часть, значит, не меньше чем на три части. Рассмотрим третью часть $P_{3}$. Из неравенств (4) и (5) следует, что $g_{03}=0$, следовательно, в третьем столбце есть непустая внутренняя клетка $(j, 3)$. Тогда по предложению 2 в клеточной форме $g_{00}+g_{j 0} \geqslant k$, откуда немедленно следует, что все вершины $A$ лежат в $Q_{j}$. Получается противоречие с зависимостью $A$ и $B$. Итак, все вершины графа, кроме $A \cup B$, лежат или в какой-то одной строчке, или в каком-то столбце. Разберем оба случая.

Если все вершины какой-то $i$-й строчки лежат в крайней клетке $(i, 0)$, то

$$
g_{i 0}>k /(p+1)
$$

Действительно, вершины этой клетки могут быть соединены только с другими вершинами этой клетки и с вершинами $B$. Если бы выписанное неравенство не выполнялось, вершины этой клетки имели бы степени не выше $k+k /(p+1)-1$, что противоречит условию. Аналогично, верен соответствующий факт для столбцов.

Итак, рассмотрим случай, когда все непустые внутренние клетки лежат в одной строке, скажем, в первой. Сюда же отнесем случай, когда непустых клеток нет вовсе. Согласно (6), верно неравенство $g_{20}>k /(p+1)$, откуда $g_{00}+g_{10}<k-k /(p+1)$. Тогда если в $j$-м столбце есть непустые внутренние клетки, то это может быть только клетка $(1, j)$, и по предложению 2 выполнено неравенство $g_{0 j}>k /(p+1)$. Если же их нет, то неравенство $g_{0 j}>k /(p+1)$ выполнено согласно (6). Суммируя эти неравенства по всем клеткам нулевой строки, приходим к противоречию с тем, что в множестве $B$ только $k$ вершин.

Теперь рассмотрим случай, когда все непустые внутренние клетки (и их не меньше одной) лежат в первом столбце. Согласно (6), верно неравенство $g_{0 i}>k /(p+1)$ при всех $i>1$. Следовательно, $g_{01}+g_{00}<k /(p+1)$. Не умаляя общности, будем считать, что в первом столбце непустой оказалась клетка $(1,1)$. По предложению 2 в клеточной форме $g_{10}>k-k /(p+1)$. Если клетка $(2,1)$ пуста, то согласно неравенству (6) выполнено неравенство $g_{20}>k /(p+1)$. Если она непуста, то, как и в случае с клеткой $(1,1)$, получаем, что $g_{20}>k-k /(p+1)$. В любом случае, приходим к тому, что размер $A$ больше $k$. Получаем противоречие.

Следствие 1. Пусть степени всех вершин графа не меньше, чем $k(p+2) /(p+1), u$ каждый представитель набора $\mathfrak{U}$ из $n$ множеств зависим с каким-то разделяючим множеством графа. Тогда в $\mathfrak{U}$-разбиении будет не более 2 невырожденных частей при $p=2$ и не более $p n-n+1$ невырожденных частей в случае $p>2$.

Доказательство. По теореме 5 каждое множество набора $\mathfrak{A}$ режет граф не более чем на $p$ частей. Отсюда и по теореме 2 получается необходимая оценка.

Замечание 4. Из результатов работы [10] вытекает частный случай этого следствия при $p=2$, но при более грубой нижней оценке на степени вершин $3 k / 2$ против доказанной в этой статье $4 k / 3$.

Замечание 5. Если не делать предположения о зависимости, то можно предложить следующую тривиальную оценку: число частей в $A$-разрезе не превосходит максимальной степени вершин $A$, а значит, и максимума степеней в графе. 


\section{Список литературы}

1. Харари Ф., Теория графов. Мир, Москва, 1973.

2. Оре О.,, Теория графов. Наука, Москва, 1968.

3. Matula D. W., $k$-Blocks and ultrablocks in graphs. J. Comb Theory, Ser. B (1978) 24, 1-13.

4. Harary F., Kodama Y., On the genus of an $n$-connected graph. Fund. Math. (1964) 54, 7-13.

5. MacLane S., A structural characterization of planar graph. Duke Math. J. (1937) 3, 460-472.

6. Tutt W. T., Connectivity in graphs. Univ. Toronto Press, Toronto, 1966.

7. Hopcroft J. E., Tarjan R. E., Dividing a graph into triconnected components. SIAM J. Comput. (1973) 2, 135-158.

8. Hohberg W., The decomposition of graphs into $k$-connected components. Discrete Math. (1992) 109, 133-145.

9. Карпов Д. В., Пастор А. В., О структуре $k$-связного графа. Записки научных семинаров ПОМИ (2000) 266, 76-106.

10. Карпов Д. В., Блоки в $k$-связном графе. Записки научных семинаров ПОМИ (2002) 293, 59-93.

11. Грэхем Р., Кнут Д., Паташник О., Конкретная математика. Мир, Москва, 1998.

12. Iverson K. E., A programming language. Wiley, New York, 1962.

Статья поступила 17.07.2003.

Переработанный вариант поступил 07.06.2004. 